\title{
Unimodality of Steady Size Distributions of Growing Cell Populations*
}

\author{
F. P. da Costa, \\ Department of Mathematics \\ Instituto Superior Técnico \\ Lisboa, Portugal
}

\author{
M. Grinfeld \\ Department of Mathematics \\ University of Strathcldye \\ Glasgow, U.K.
}

\author{
J. B. McLeod \\ Department of Mathematics \\ University of Pittsburgh \\ Pittsburgh, PA
}

To the memory of Tosio Kato, in admiration of his work and recalling a very happy collaboration.

\begin{abstract}
We consider an equation for the evolution of growing and dividing cells, and show, using a result of Kato and McLeod, that the probability density function for the stationary size distribution is necessarily unimodal.
\end{abstract}

\section{Introduction}

In $[1,2]$ Hall and Wake consider the evolution of a population of growing and dividing cells. If we let $n(x, t) d x$ be the number at time $t$ of cells of sizes between $x$ and $x+d x$, then $n(x, t)$ satisfies the following hyperbolic functional partial differential equation:

${ }^{*}$ Published in J. Evol. Equ. 1, 405-409 (2001). 


$$
n(x, t)_{t}=-(g(x) n(x, t))_{x}-b(x) n(x, t)+\alpha^{2} b(\alpha x) n(\alpha x, t), x \in \mathbb{R}_{+} .
$$

In this equation a mother cell of size $x$ divides into $\alpha>1$ (usually $\alpha=2$ ) daughter cells of the same size $x ; g(x)$ is the growth rate, and $b(x)$ is the division rate, of a cell of size $x$.

Note that there is no mortality of cells, so the reasonable boundary conditions for (1.1) are

$$
g(0) n(0, t)=0 \text { and } \lim _{x \rightarrow \infty} g(x) n(x, t)=0 \quad \forall t>0 .
$$

Denote the right-hand side of (1.1) by $A(n)$. Below we shall assume that $b(x) \geq 0, g(x) \geq 0$ for all $x \in \mathbb{R}_{+}$and that $b(x) / g(x) \in L^{1}\left(\mathbb{R}_{+}\right)$. Defining

$$
h(x)=\exp \left(-\int_{0}^{x} \frac{b(s)}{g(s)} d s\right)
$$

using the machinery developed in [4] and a result of [1], we have the following proposition:

Proposition 1 1. (1.1)-(1.2) generates a semiflow on the space

$$
X=\left\{u \in C\left(\mathbb{R}_{+}\right)\left|\sup _{x \in \mathbb{R}_{+}} \frac{g(x)}{h(x)}\right| u(x) \mid<\infty\right\} .
$$

2. The semiflow preserves the cone of non-negative functions in $X$.

3. There is a unique eigenvalue $\lambda>0$ for which the operator $A$ has a nonnegative eigenfunction $y(x)$; furthermore $y(x)$ is positive for all $x \in(0, \infty)$.

The key observation is that the change of variable $n=h u / g$ transforms (1.1) into a problem in which a generator of a strongly continuous semigroup is perturbed by a bounded operator. [4] treat the case of $\alpha=2$ and of cells of non-zero minimal size and finite maximal size, but the arguments go through with minor changes. Positivity of $y(x)$ for non-zero $x$ follows from the arguments of [1] for the case of constant $b(x)$ and $g(x)$. Note that if we let $N(t)$ be the total cell population, $N(t)=\int_{0}^{\infty} n(x, t) d x$, we have that 
$\lambda$ is the growth rate of $N(t)$, that is, $N(t)=N(0) e^{\lambda t}$, so that (1.1) is only applicable to exponentially growing populations.

It is the eigenfunction $y(x)$ that we are interested in. It has the interpretation of the probability density function describing the stationary size distribution (SSD). Hence we supplement the equation it has to satisfy,

$$
(g(x) y(x))^{\prime}+\lambda y=-b(x) y(x)+\alpha^{2} b(\alpha x) y(\alpha x),
$$

with the conditions

$$
y(x) \geq 0 \text { for all } x \in[0, \infty)
$$

and the normalization condition (since $y(x)$ is a probability distribution)

$$
\int_{0}^{\infty} y(x) d x=1
$$

Obviously, to be able to determine $y(x)$ we need to know $\lambda$. There are two cases where the value for $\lambda$ can be worked out explicitly; these are the cases $b(x)=\beta$ and of $g(x)=\gamma x$ with $b(x)$ growing superlinearly at infinity. In the first case by integrating (1.3) we have

$$
\lambda=(\alpha-1) \beta .
$$

In the second case we have that $\int_{0}^{\infty} g(x) y(x) d x$ is finite, and multiplying (1.3) by $x$ and integrating we have

$$
\lambda=\frac{\int_{0}^{\infty} g(x) y(x) d x}{\int_{0}^{\infty} x y(x) d x},
$$

so that in this case $\lambda=\gamma$.

The simplest interesting case of (1.3) arises if we assume that $g(x)=1$ and $b(x)=\beta$, a positive constant. Then (1.3) becomes

$$
y^{\prime}(x)=-\alpha \beta y(x)+\alpha^{2} \beta y(\alpha x), y^{\prime}(x)=-\alpha \beta y(x)+\alpha^{2} \beta y(\alpha x),
$$

subject to (1.4) and (1.5). Note that by integrating (1.7) between zero and infinity and using (1.5), we immediately have that $y(0)=0$. Equations of the form (1.7) have been described fairly completely in [3]; that paper is extensively used in [1], which also concentrates on (1.7). 
Looking at the pictures of $[1,2]$ one observes that all the SSD functions $y(x)$ are unimodal. The object of the present note is to give a proof of this fact. We first prove the result for the (biologically unrealistic) case of constant $g(x)$ and $b(x)$ and then show how this entails unimodality for reasonable choices of $g(x)$ and $b(x)$, such as, for example, $g(x)=\gamma x$ and $b(x)=\beta x^{r}$ (here $\gamma, \beta$, are positive constants, $r>1$ ). Since unimodality of the SSD is a necessary consequence of this type of model, deviation from it in experimental situations must indicate that a more sophisticated model for the dynamics of the cell population is required. We also note that the solution $N(0) \exp (\lambda t) y(x)$ in the case of $g(x)=\gamma x$ does not have good attractivity properties; see [4].

\section{Main Result}

Below we denote by $y(x)$ the SSD solution of (1.7). First of all, we prove the following elementary results:

Lemma 2 If $y(x)$ has a minimum, it must have an infinite number of such minima.

Proof. Assume on the contrary that there is a finite number of minima. Note that if $x_{0}$ is the last point of minimum for $y(x)$,

$$
y\left(\alpha x_{0}\right)=\frac{1}{\alpha} y\left(x_{0}\right),
$$

so that at $\alpha x_{0}>x_{0}$ we have that $y\left(\alpha x_{0}\right)<y\left(x_{0}\right)$. If $y(x)$ has a minimum at $x_{0}, y^{(2 m)}\left(x_{0}\right)>0$ for some positive integer $m$. Below we give the argument for $m=1$; the degenerate case follows along similar lines. If $m=1$, it suffices to differentiate the equation (1.7) at $x=x_{0}$ once (in the general case it has to be done $2 m-1$ times). Thus we have

$$
y^{\prime \prime}\left(x_{0}\right)=\alpha^{3} \beta y\left(\alpha x_{0}\right) .
$$

Hence $y^{\prime}\left(\alpha x_{0}\right)>0$, which implies that there is a minimum at some $x_{*}>x_{0}$, leading to a contradiction.

Lemma 3 If $y(x)$ has an infinite number of minima, these cannot accumulate at a finite point. 
Proof. Let $x_{0}$ be the last accumulation point. Then by the above argument there must exist a minimum between $x_{0}$ and $\alpha x_{0}$.

Now, using Lemmas 2 and 3 we can prove

Theorem $4 y(x)$ is unimodal.

Proof. Kato and McLeod [3] (see Theorems 3 and 9 there) discuss the equation

$$
y^{\prime}(x)=A y(\theta x)+B y(x),
$$

which is the same as (1.7) under the identification $\theta=\alpha, B=-\alpha \beta, A=$ $\alpha^{2} \beta$. Hence the parameter $\kappa$ of Theorem 3 in [3], given by $\kappa=\operatorname{Re} k_{0}$, where $k_{0}$ is any solution of

$$
k=\frac{\log (-B / A)}{\log \theta},
$$

becomes

$$
\kappa=\operatorname{Re}\left(\frac{\log \left(\alpha \beta /\left(\alpha^{2} \beta\right)\right)}{\log \alpha}\right)=-1
$$

Hence by Theorem 9 of [3], any solution of (1.7) which is $o\left(x^{\kappa}\right)=o\left(x^{-1}\right)$ as $x \rightarrow \infty$ is necessarily a multiple of

$$
y_{0}=e^{-\alpha \beta x}\left[1+\sum_{n=1}^{\infty}(-1)^{n} \frac{\left(\alpha^{2} \beta\right)^{n} \exp \left\{\alpha \beta\left(1-\alpha^{n}\right) x\right\}}{(-\alpha \beta)^{n}(1-\alpha)\left(1-\alpha^{2}\right) \cdots\left(1-\alpha^{n}\right)}\right] .
$$

It is clear that $y_{0}=O(\exp (-\alpha \beta x))$ for large $x$, and hence from (1.7) it is obvious that $y_{0}$ is ultimately monotone decreasing, and so therefore is any solution of (1.7) that is $o\left(x^{-1}\right)$. Since we have by Lemmas 2, 3 that any non-unimodal solution has necessarily an infinite number of minima going off to infinity, we see that any solution of (1.7) that is $o\left(x^{-1}\right)$ is necessarily unimodal. However, since $y(x)$ is an SSD (in fact the main result of [1] is the computation of $C$ such that $C y_{0}(x)$ is the SSD), by the normalization condition it has to be $o\left(x^{-1}\right)$. As discussed in [2], it is not biologically realistic to assume that the growth rate $g(x)$ and the division rate $b(x)$ of a cell of size $x$ are independent of $x$. [2] discuss the case of $g(x)=\gamma x$ and $b(x)=\beta x^{r}$, where $\gamma, \beta, r$ are all positive constants. [2] show that in this case the SSD can be written in the form

$$
y(x)=C \frac{1}{x^{2}} Y_{0}\left(x^{r}\right),
$$


where $Y_{0}$ is a solution of the same form as $y_{0}$ of $(2.9)$, i.e. $Y_{0}(x)$ satisfies equation (2.8) for some choice of $\theta, A>0$ and $B<0$. Hence all the arguments of Theorem 4 hold, and the SSD is unimodal.

\section{Acknowledgments}

MG expresses his gratitude to Prof. G. C. Wake for introducing him to the problem discussed in this note and to the Leverhulme Trust for financial support. FPC was supported in part by FCT (Portugal) through program POCTI.

\section{References}

[1] A. J. Hall and G. C. Wake, A functional differential equation arising in the modelling of cell growth, J. Austral. Math. Soc. Ser B 30 (1989), 424-435.

[2] A. J. Hall and G. C. Wake, Functional differential equations determining steady size distributions for populations of cells growing exponentially, J. Austral. Math. Soc. Ser B 31 (1990), 434-453.

[3] T. Kato and J. B. McLeod, The functional differential equation $y^{\prime}(x)=$ $a y(\lambda x)+b y(x)$, Bull. Amer. Math. Soc. 77 (1971), 891-937.

[4] The Dynamics of Physiologically Structured Populations, J. A. J. Metz and O. Diekmann, eds. Springer-Verlag, Berlin 1986. 